Journal of the Operations Research

Society of Japan

Vol. 24, No. 2, June 1981

\title{
FREE BOUNDARY VALUE PROBLEM AND INVARIANT IMBEDDING
}

\author{
Kuo-ming Wang \\ National Tsing Hua University
}

\author{
E. Stanley Lee \\ Kansas State University
}

(Received November 25, 1980)

\begin{abstract}
Many practical engineering design problems are the free boundary-value type problem. In this work, free boundary-value problem is solved by combining method of characteristics and invariant imbedding approach. To show the effectiveness of this approach, the length of a chemical reactor with axial mixing process is estimated by the proposed approach. It is shown that with reasonable step size, we can get very accurate length of a chemical reactor process.
\end{abstract}

\section{Introduction}

One of the most important problems in the process industry is the designing of equipments. For example, on the conditions that the raw material and the desired products are given, we are required to estimate (design) the size of the equipment to produce these desired products for the given raw material. These design problems form typical boundary value problems with the raw material and the given products as the boundary conditions. Some examples of design problems are:

design the size (length) of the distillation columns, design the size (length) of a tubular reactor, determine the time (length) required for a particle to move from one position to another position, determine the time required for a certain chemical reaction process, determine the time required for the concentration of a drug to reach a certain level at certain part of our body.

In this work, the size (length) of a chemical reactor with axial mixing mode1 [2] is estimated by the proposed approach. 
2. The Problem of Estimating the Length of a Process $(T)$

Consider a system whose behavior can be represented by a nonlinear second order differential equation [2]

$$
d^{2} \times / d t^{2}=P_{1}(d x / d t)+P_{2} x^{2}
$$

with input data

$$
\begin{aligned}
& x(0)=C_{1} \\
& x^{\prime}(0)=C_{2}
\end{aligned}
$$

and

$$
x(T)=C_{3}
$$

The problem is to calculate the length of the process, T, by using Eqs. (1)-(3) and the proposed approach.

3. The Invariant Imbedding Approach

In order to illustrate, we will rewrite Eqs. (1)-(3)

$$
\begin{aligned}
& d x / d t=y=f_{1} \\
& d y / d t=P_{1} \cdot y+P_{2} \cdot x^{2}=f_{2}
\end{aligned}
$$

with input data

$$
\begin{aligned}
& x(0)=C_{1} \\
& y(0)=C_{2} \\
& x(T)=C_{3}
\end{aligned}
$$

By invariant imbedding concept as mentioned in Ref. 2, we can formulate an invariant imbedding equation for $T$ as a function of $C_{1}$ and $C_{2}$ :

$$
T\left(C_{1}, C_{2}\right)=\Delta+T\left(C_{1}+\Delta \cdot f_{1}, C_{2}+\Delta \cdot f_{2}\right)
$$

where $T\left(C_{1}, C_{2}\right)$ is the length of the process represented by Eqs. (4)-(6) when the process starting at time $a$ and with $x(a)=C_{1}$ and $y(a)=C_{2}$.

Expanding Eq. (7) by Taylor series formula, we have

$$
T\left(C_{1}, C_{2}\right)=\Delta+T\left(C_{1}, C_{2}\right)+\partial T / \partial C_{1} \cdot \Delta \cdot f_{1}+\partial T / \partial C_{2} \cdot \Delta \cdot f_{2}+o(\Delta)
$$

Rearrange Eq. (8) and as $\Delta \rightarrow 0$, we obtain

$$
\partial T / \partial C_{1} \cdot f_{1}+\partial T / \partial C_{2} \cdot f_{2}=-1
$$




\section{The Method of Characteristics}

Various techniques have been used to solve Eq. (9). In this work, we shall use the method of characteristics. The characteristic equation of Eq. (9) is [1]

$$
d C_{1} / f_{1}=d C_{2} / f_{2}=-d T / 1
$$

From the left-half of Eq. (10), we have

$$
d C_{2} / d C_{1}=f_{2} / f_{1}=P_{1}+P_{2} \cdot C_{1}^{2} / C_{2}
$$

Solve Eq. (11) either by analytical or numerical methods, we can obtain

$$
C_{2}=f\left(C_{1}\right)
$$

From the right-half of Eq. (10), we have

$$
d C_{1} / f_{1}=-d T / 1
$$

Integrate Eq. (12), we have

$$
T\left(C_{1}, C_{2}\right)=-\int_{0}^{C_{1}} d C_{1} / f_{1}+\psi
$$

According to definition of $T\left(C_{1}, C_{2}\right)$, we see that

$$
T\left(X\left(I^{\prime}\right), Y(T)\right)=0
$$

Thus, Eq. (13) becomes

$$
-\int_{0}^{X(T)} d c_{1} / f_{1}+\psi=0
$$

From Eq. (3) and Eq. (14), we obtain

$$
\psi=\int_{0}^{C_{3}}\left(1 / f_{1}\right) d C_{1}
$$

Substituting Eq. (15) into Eq. (13), we have

$$
T\left(C_{1}, C_{2}\right)=+\int C_{1}^{C_{3}}\left(1 / f_{1}\right) d C_{1}
$$

5. Numerical Example

Consider the chemical reaction [2]

$$
\mathrm{A}+\mathrm{A} \rightarrow \mathrm{B}
$$

which is taking place in a homogeneous tubular flow chemical reactor with 
axial mixing. Assume that the change of volume in the reactor is negligible, the following equation can be established easily by the use of material balance on reactant A [2]

$$
\left(1 / N_{P}\right) \cdot d^{2} x / d t^{2}-d x / d t-R \cdot x^{2}=0
$$

or

$$
d^{2} x / d t^{2}=N_{P_{e}} \cdot d x / d t+N_{P_{e}} \cdot R \cdot x^{2}
$$

with input data

$$
\begin{aligned}
& x(0)=C_{1} \\
& x^{\prime}(0)=C_{2} \\
& x(T)=C_{3}
\end{aligned}
$$

The notation of the above model is the same as cited in reference 2 . The problem is to estimate the length of the process, $T$, by using the proposed approach.

\section{Computational Procedures}

Eq. (17) is a second order nonlinear differential equation which can be transformed into two first order differential equations

$$
\begin{aligned}
& d x / d t=y \\
& d y / d t=N_{P_{e}} \cdot y+N_{P_{e}} \cdot R \cdot x^{2}
\end{aligned}
$$

with input data

$$
\begin{aligned}
& x(0)=C_{1} \\
& y(0)=C_{2} \\
& x(T)=C_{3}
\end{aligned}
$$

According to Eqs. (7)-(9), we obtain the invariant imbedding equation for $T\left(C_{1}, C_{2}\right)$ as follows

$$
\partial T / \partial C_{1} \cdot C_{2}+\partial T / \partial C_{2} \cdot\left(N_{P_{e}} \cdot C_{2}+N_{P} \cdot R \cdot C_{1}^{2}\right)=-1
$$

According to Eq. (10), the characteristic equation for Eq. (23) is

$$
d C_{1} / C_{2}=d C_{2} /\left(N_{P_{e}} \cdot C_{2}+N_{P_{e}} \cdot R \cdot C_{1}^{2}\right)=-d T / 1
$$


From the first term of Eq. (24), we have

$$
d C_{2} / d C_{1}=\left(N_{P} \cdot C_{2}+N_{P} R \cdot C_{1}^{2}\right) / C_{2}
$$

with

$$
C_{2}\left(C_{1}=X(0)\right)=Y(0)
$$

From the first and third terms of Eq. (24), we have

$$
d C_{1} / C_{2}=-d T / 1
$$

Thus, according to Eq. (16), we obtain

$$
T\left(C_{1}, C_{2}\right)=\int_{C_{1}}^{C_{3}}\left(1 / C_{2}\right) d C_{1}
$$

Therefore, with given values of $X(0), X^{\prime}(0)$, and Eqs. (25) and (26), the $C_{2}$ values at various $C_{1}$ values can be obtained. Substituting the $C_{2}$ values into Eq. (28), we can calculate ' $T$ ' value with given $C_{1}$ and $C_{3}$.

7. Numerical Results

The following numerical values are used in the calculation:

$$
\begin{aligned}
& X(0)=C_{1}=0.83129 \\
& Y(0)=X^{\prime}(0)=C_{2}=-1.0122 \\
& X(T)=C_{3}=0.38737 \\
& N_{P_{e}}=6 \\
& R=2
\end{aligned}
$$

Following the numerical procedures listed in section 6 , we obtain

$$
T=1.03
$$

8. Discussion and Conclusion

The length of the process, $T$, obtained by this approach is $T=1.03$ which is very close to the true value $T=1$. We can get more accurate results by further reducing the value of step size, $\Delta C_{1}$.

Although only a second order nonlinear differential equation is solved in this work, this approach can be extended to more higher order problem. 


\section{REFERENCES}

[1] Abbott, M. B.: An Introduction to the Method of Characteristics. American Elsevier, New York, 1966.

[2] Lee E. S.: Quasilinearization and Invariant Imbedding. Academic Press, 1968.

Kuo-ming WANG: Department of Industrial

Engineering, National Tsing Hua

University, Hsinchu, Taiwan, China. 